\title{
Seroprevalence of Dengue in Antenatal and Paediatric Patients - In a Tertiary Care Hospital, Puducherry
}

\author{
Vani Gopal*, Aruna and P. Sujatha \\ Rajiv Gandhi Govt. Women and Children Hospital, Ellaipillaichavady, Puducherry, India \\ *Corresponding author
}

\section{A B S T R A C T}

\begin{tabular}{|c|}
\hline Keywords \\
\hline $\begin{array}{l}\text { Dengue, Aedes aegypti } \\
\text { mosquito, NS1 Ag \& IgM } \\
\text { ELISA, Seroprevalence, } \\
\text { Antenatal and Paediatric } \\
\text { patients }\end{array}$ \\
\hline Article Info \\
\hline $\begin{array}{l}\text { Accepted: } \\
\text { 04 May } 2018 \\
\text { Available Online: } \\
\text { 10 June } 2018\end{array}$ \\
\hline
\end{tabular}

\section{Introduction}

Dengue is a vector borne arboviral disease caused by dengue virus belonging to Flaviviridae family (DEN V1-4 serotypes) (Mustafa et al., 2015). It has become endemic in India over two centuries. Dengue virus infection is transmitted by Aedes aegypti mosquito and is prevalent in tropical and subtropical regions (Jhansi Charles et al., 2015).

It exhibits a wide spectrum of clinical manifestations ranging from asymptomatic to severe clinical manifestation such as dengue shock syndrome (Srinivasa Rao Mutheneni et
Dengue is a vector borne arboviral disease caused by dengue virus, and it is transmitted by Aedes aegypti mosquito. It is endemic in almost all parts of India for the past two decades. Recently there was dengue outbreak in the year 2017 where southern parts of India were most affected. This study is carried out in a tertiary care child and maternity hospital, Puducherry, to study the seroprevalence rate of dengue among antenatal and peadiatric age group during the time period January 2017 to December 2017. NS1Ag ELISA and IgM Microlisa tests were done to diagnose dengue infection. The Prevalence rate in Antenatal cases and Paediatric patients was $16.39 \%$ and $31.36 \%$ respectively. Peak prevalence was observed in the age group 9-12 years and lowest was seen in the age group 0-3 years among paediatric patients. The peak incidence of dengue infection was observed in the months of August, September and October and declined thereafter. 
Studies regarding dengue prevalence particularly in antenatal and paediatric patients are not available. Hence this study will show the prevalence rate in Antenatal and Paediatric patients in a Tertiary care hospital, Puducherry.

\section{Aims and Objectives}

To estimate the prevalence of Dengue during the year 2017, in Antenatal and Paediatric patients in a Tertiary care hospital, Puducherry.

To estimate the age-wise and sex-wise distribution of dengue infection during the year 2017.

To estimate the month-wise distribution of dengue infection and peak level of incidence during the year 2017.

\section{Materials and Methods}

This is a retrospective study conducted from January 2017-december2017 at Rajiv Gandhi Govt. Women and Children Hospital, Puducherry. Laboratory reports of the antenatal and paediatric patients who had been sent to our lab with history of fever, congestion of eyes, rashes, petechial spots were collected.

\section{Diagnosis of dengue}

Diagnosis of Dengue was done by detecting Dengue non-structural protein (NS1 antigen) and Dengue specific $\operatorname{IgM}$ antibody by Enzyme-Linked Immunosorbent assay (ELISA). Dengue NS1 antigen kit was procured by National Vector Borne Disease Control Programme (NVBDCP) (Gupta et al., 2006). Dengue specific IgM antibody was detected by IgM antibody Capture (MAC ELISA) kits provided by National Institute of Virology (Pune). These kits have sensitivity of
$99.5 \%$ and specificity of $100 \%$. The test procedure was done with the serum samples using the Kit Manual provided along with the kits. Both positive and negative controls were used in each run. The absorbance value was read between $450 \mathrm{~nm}$ and $630 \mathrm{~nm}$ using ELISA reader manufactured by Erba Diagnostics, Lisascan.

\section{Results and Discussion}

A total of 744 samples of antenatal cases with history of fever were tested for Dengue NS1Ag and IgM. Out of 744 samples, 122 samples were found to be positive (16.39\%) out of which 112 cases were NS1Ag positive and 12 samples were IgM positive. Among the 1272 paediatrics samples, 399 (31.36\%) were diagnosed to have Dengue infection.

Paediatric patients of age group 9-12 years showed highest prevalence (44.9\%) of Dengue infection and the age group of 0-3years showed lowest prevalence (8.5\%). Among others, 3-6 years and 6-9 years groups had the prevalence rate of $22.3 \%$ and $24.3 \%$ respectively.

Dengue positivity rate started increasing from the month of July 2017 and the peak incidence was found in the months of September and October followed by a gradual decline from November onwards.

The peak incidence was found to be in the months of August to October and gradually decreased thereafter.

Dengue is one of the major health threats in India for the past two decades and the incidence increased 30 fold between 1960 and 2010. (Vaddadi Srinivas and Vaddadi Radha Srinivas, 2015) There was a dengue outbreak in the year 2012, which affected predominantly regions around Kolkata. (http://www.nvbdcp.gov.in/dencd.html) 
Another outbreak occurred during the year 2017, which was prevalent in southern parts of India including Kerala, Karnataka, Puducherry and Tamilnadu. This study revealed the data related to dengue infection among antenatal and paediatric patients during 2017 outbreak occurred in Puducherry territory.

The overall prevalence rate of dengue infection in both antenatal and paediatric patients was $25.8 \%$, out of which $16.4 \%$ was contributed by antenatal cases. Very few studies are available regarding the prevalence rate of Dengue infection in Indian Antenatal cases and most of the studies are on general population. In a study during the year 2013, the rate of infection in antenatal cases was only $0.72 \%$ (Pavanaganga et al., 2017). It's a retrospective study and the less prevalence may be due to non-seasonal outbreak during that period. Another study in Malaysia showed $2.5 \%$ prevalence rate of pregnancy in the year 2008 (Peng Chiong Tan et al., 2008)
Our study showed that the prevalence rate dengue infection in Children was 31.3\%. Highest prevalence rate was seen in the age group of 9-12yrs (45\%) followed by 6-9 years (24\%). In a study by Agarwal et al., (1998) the prevalence rate of dengue in children was $20 \%$ (Agarwal and Chandra, 1998). In another study by Kabilan et al., (2003), common age group affected were in 5-9 years. The prevalence rate is high in children which may be due to the habit of spending more time in outdoors which may expose them to frequent mosquito bites.

In Indian study conducted among the children of 5-10 years revealed prevalence of $59 \%$. In that study, Mumbai showed highest prevalence of $80 \%$ and lowest (23\%) in West Bengal. In an Indonesian population, the prevalence of Dengue was found to be $69.4 \%$ in the $1-4$ years old, $65.4 \%$ in the 5-9 years old and $83.1 \%$ in the 10-14 years old in 2017 (Prayitno et al., 2017) (Fig. 1-5).

Fig.1 Dengue positive among fever cases

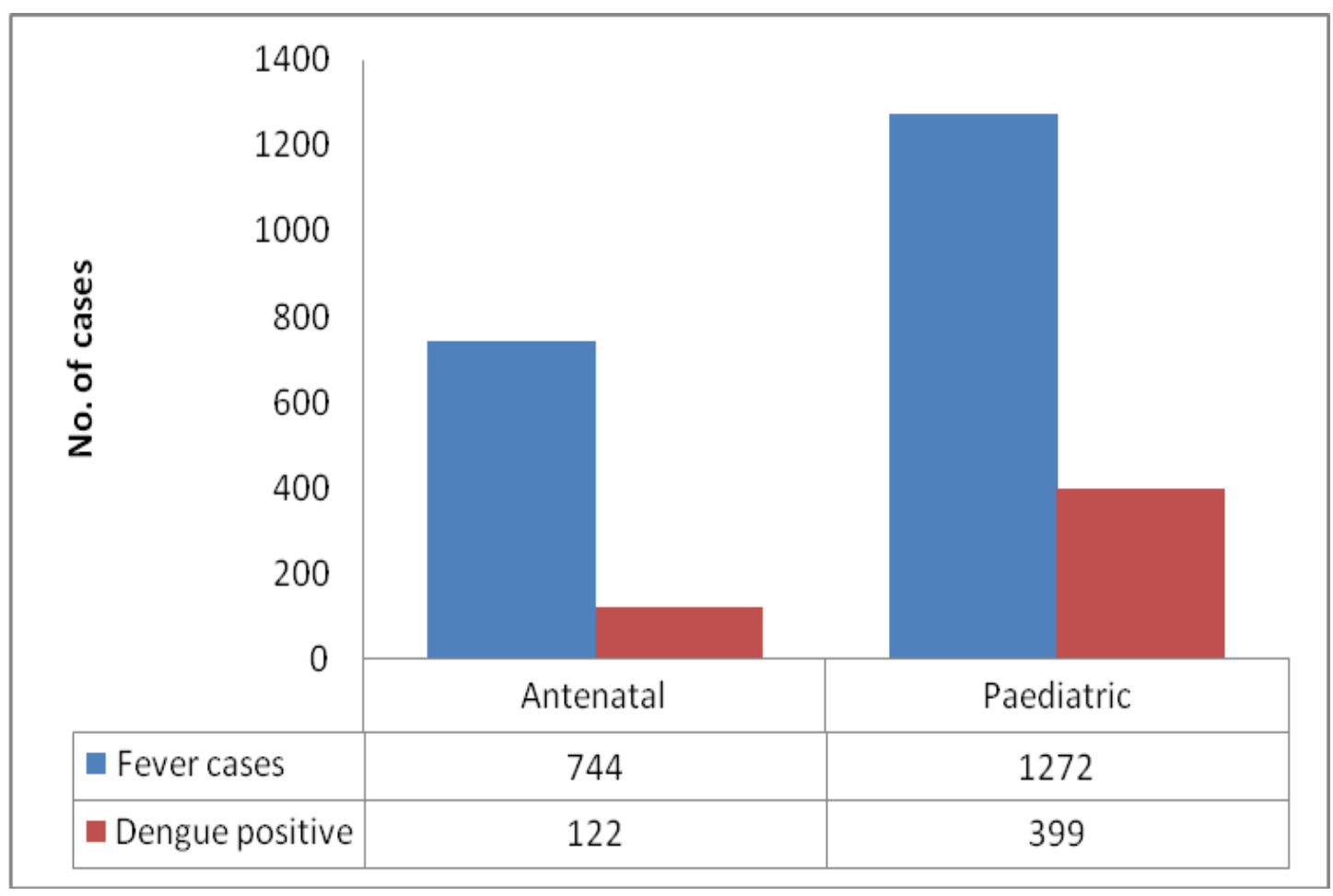


Fig.2 NS1 Ag and IgM positivity among fever cases

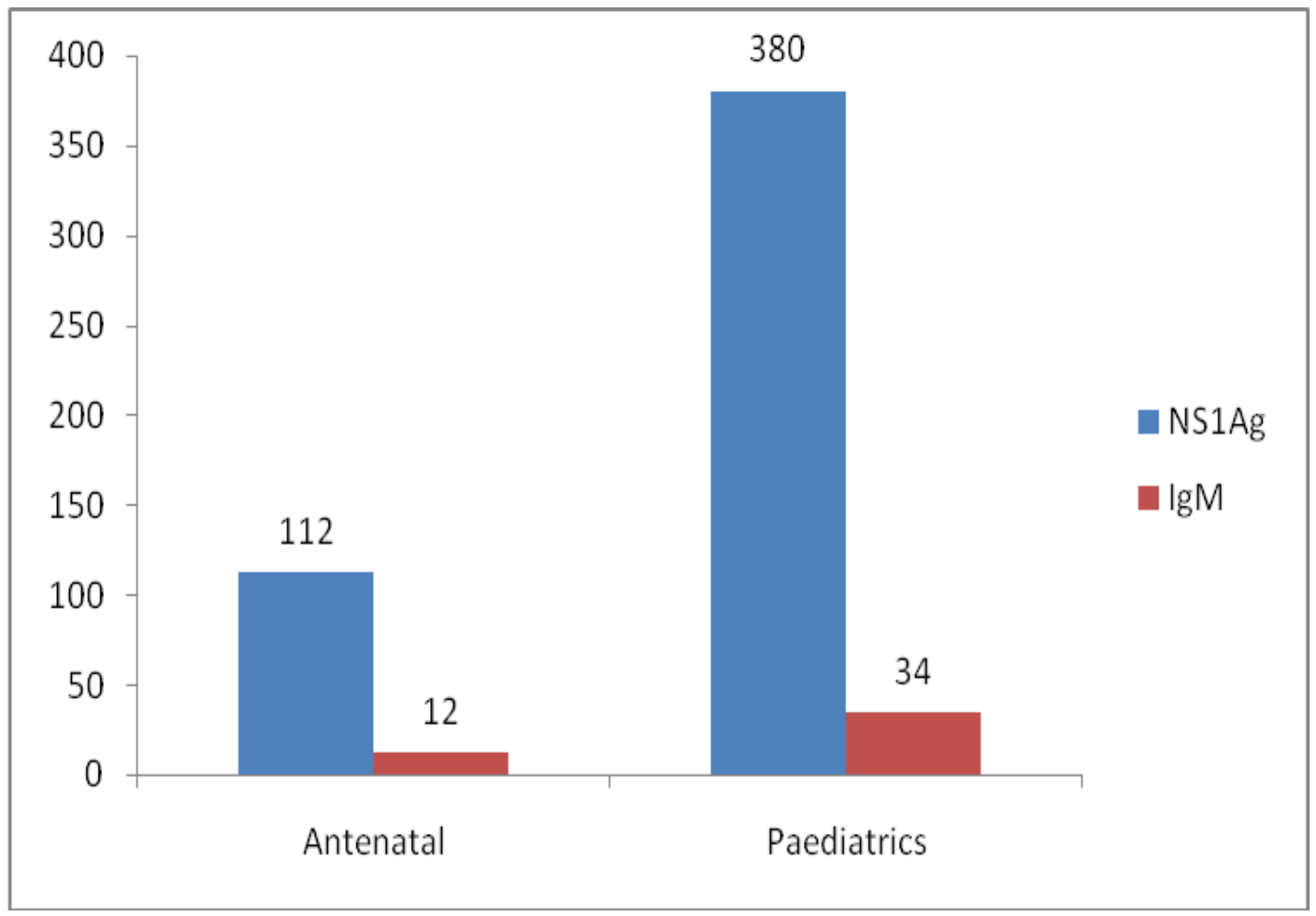

Fig.3 Age wise distribution of dengue infection among paediatric cases

\section{Age wise distribution of Dengue among paediatric cases}

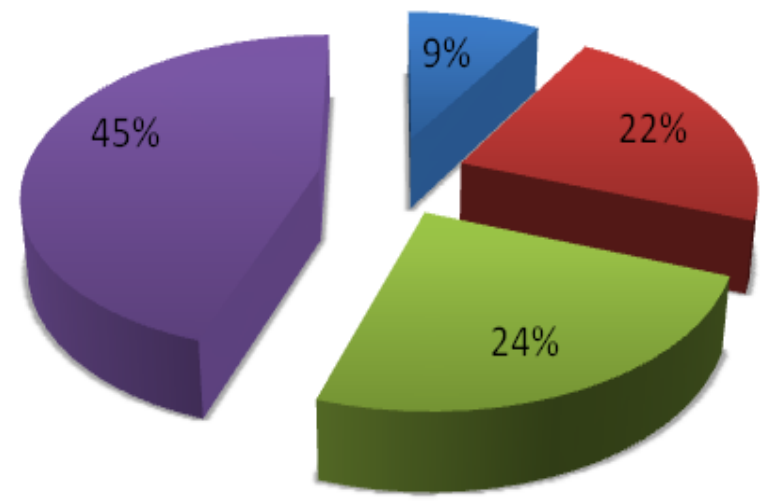

$\square 0-3$ years $\square 3-6$ years $\square-9$ years $\square 9-12$ years 
Fig.4 Month-wise distribution of dengue among paediatric patients

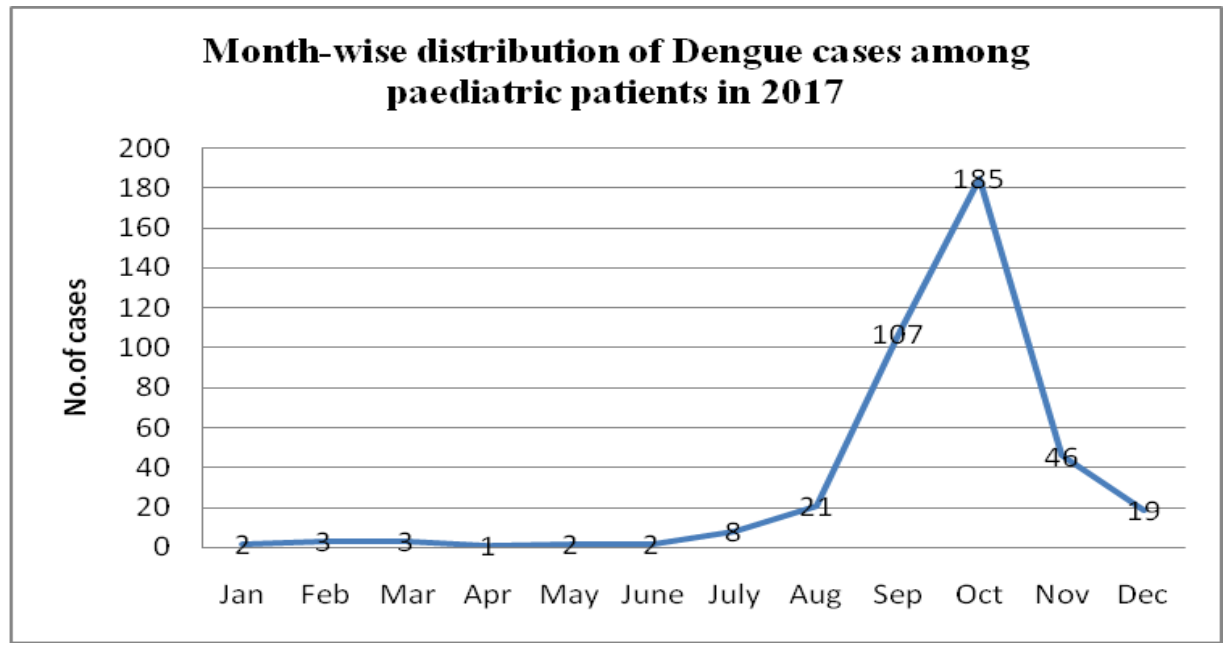

Fig.5 Month-wise distribution of dengue among antenatal patients

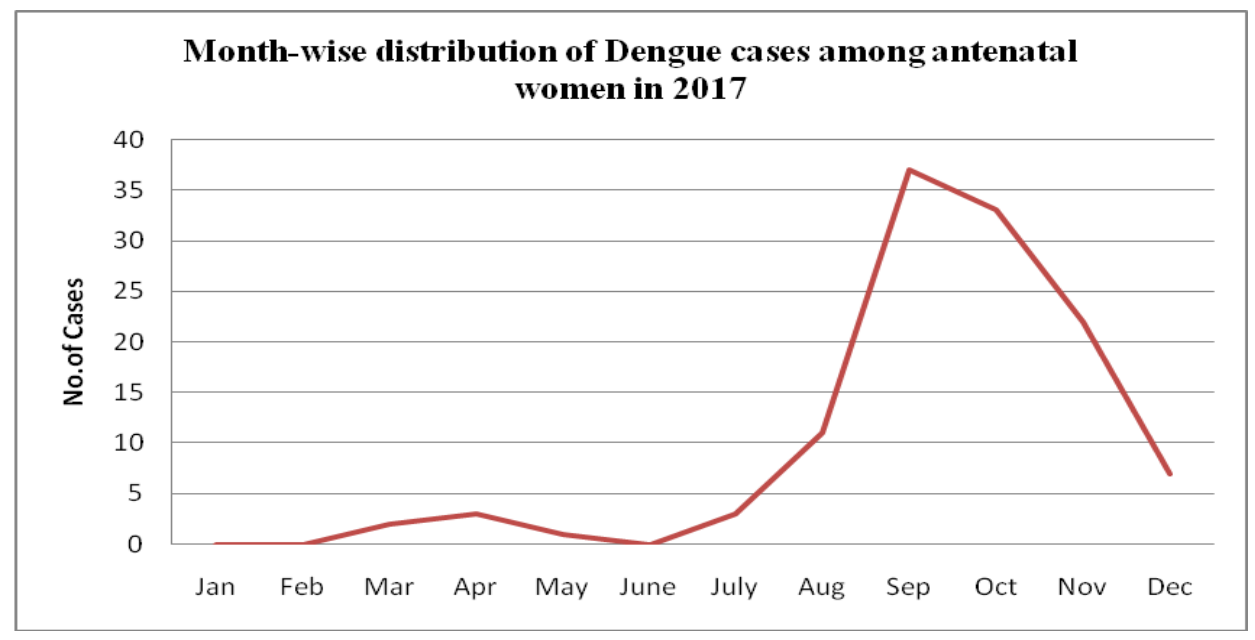

Among the antenatal dengue positive cases, 112 samples were NS1 positive and 12 samples showed IgM positive and in Paediatric samples 380 samples were NS1 antigen positive and 34 samples IgM positive. NS1 antigen can be detected from Day 1 of infection and IgM from day 5 of illness. Anyhow both the parameters indicate primary infection. In this study, the peak incidence of Dengue cases was during the period September and October.

This finding was in consistent other studies by (Ashwini Kumar et al., 2010). This may be due to post monsoon period which favours the mosquito breeding.
Dengue is a major health threat and it had become an endemic disease in India over two centuries. Recently Dengue outbreak was reported in 2017.

Dengue is a major health threat and it has become endemic in India over two centuries. It is more prevalent during post monsoon period which favours mosquito breeding. So proper sanitation measures and public awareness regarding cleanliness and to avoid stagnation of water will prevent the dengue outbreak. As the children are more vulnerable to dengue infection proper protective clothing and mosquito repellents should be advised while 
playing in the outdoor. As the dengue infection can be diagnosed from day 1 of infection, public awareness should be created through media or posters to visit nearby hospitals so that early diagnosis and treatment will prevent morbidity and mortality.

\section{Acknowledgement}

We Acknowledge NVBDCP (National Vector Borne Disease Control Programme) Programme Manager, for providing ELISA kits for this study.

\section{Conflicts of Interest}

Institutional ethical committee clearance was got and the authors report that there is no conflict of interest in this work.

\section{References}

Agarwal A, and Chandra J, An epidemic of dengue hemorrhagic fever and dengue shock syndrome in children in Delhi, Indian paediatrics 1998; 35(8): 727-32.

Ashwini Kumar, Chythra R Rao, Vinay Pandit et al., Clinical manifestations and trend of Dengue cases Admitted in a Tertiary care Hospital, Udupi District, Karnataka, Indian J Community Med, 2010, 35(3): 386-90.

Government of India, Health and Family Welfare department, National Vector Borne Disease Control Programme, (NVBDCP): Dengue cases and deaths in the country since 2007. e2012. Available from: http://www.nvbdcp. gov.in/dencd.html, accessed on December 5, 2012.

Gupta E, Dar L, Kapoor G, Broor S. The changing epidemiology of dengue in Delhi, India. Virol J. 2006; 3: 92-96.
Jhansi Charles, A. Ramesh, Anand Janagond et al., Study of prevalence of dengue infection in a rurally situated tertiary care hospital, Tamilnadu, Journal of dental and medical sciences 2015; 14: 32-36.

Mustafa MS, Rasotgi V, Jain S et al., Discovery of fifth serotype of dengue virus (DEN5): a new public health dilemma in dengue control. Med J Armed Forces India 2015; 71: 67-70.

Pavanaganga, A., MPA Sailakshmi, BR Rekha, Dengue fever during Pregnancy: Maternal and Fetal complications, Journal South Asian Federation of Obstetrics and Gynaecology 2017; 9(2): 88-91

Peng Chiong Tan, Geetha Rajasingam, Shamala Devi and Siti Zawiah Omar. Dengue Infection in Pregnancy, Prevalence, Vertical Transmission, and Pregnancy Outcome. M. Med. 111(5); 2008: 11111117.

Prayitno, A, Taurel AF et al., Dengue seroprevalence and force of primary infection in a representative population of urban dwelling Indonesian children. PLoS Negl Trop Dis. 2017 Jun 15; 11(6): e000562.

Ramakrishnan SP, Geljand HM, Bose PN et al., The epidemic of acute haemorrhagic fever, Calcutta, 1963; epidemiological inquiry. Indian J Med Res 1964; 52: 633-650.

Srinivasa Rao Mutheneni, Andrew P Morse, Cyril Caminade et al., Dengue burden in India: recent trends and importance of climatic parameters: Emerging microbes and infections 2017; 57: 1-10

Vaddadi Srinivas and Vaddadi Radha Srinivas. "Dengue Fever: A Review Article". Journal of Evolution of Medical and Dental Sciences 2015; Vol. 4, Issue 29, April 09; Page: 5048-5058, DOI: 10.14260/jemds/ $2015 / 736$.

\section{How to cite this article:}

Vani Gopal, Aruna and Sujatha, P. 2018. Seroprevalence of Dengue in Antenatal and Paediatric Patients - In a Tertiary Care Hospital, Puducherry. Int.J.Curr.Microbiol.App.Sci. 7(06): 667-672. doi: https://doi.org/10.20546/ijcmas.2018.706.077 